\title{
Numerical Techniques at Millimeter Wave Frequencies for Wireless Body Area Networks-A Review
}

\author{
Khaleda Ali
}

\begin{abstract}
Millimeter wave frequencies offering higher bandwidth, reliable performance and unlicensed use has become an alluring option for the future of wireless body area networks. This paper discusses about the scopes and limitations of different numerical techniques at millimeter wave frequencies for analyzing propagation in such networks. It is observed that at this spectrum, Finite difference time domain technique though computationally expensive, can be the most ideal tool for accurate channel estimation. For efficient use of resources a customized version of FDTD has also been presented in this paper. Convergence test of the FDTD algorithm has also been performed to evaluate the optimum discretization step which ensures accuracy and efficiency.
\end{abstract}

Index Terms-Body Area Network, Finite Difference Time Domain Technique Millimeter Wave, On-Body Propagation.

\section{INTRODUCTION}

Recent advances in wireless communications and an essential need for remote health monitoring have triggered the rapid expansion of body centric wireless communication systems (BCWC) [1]. To sustain this enormous growth of wearable industry, reliable and efficient systems are being developed working at various frequency spectra. In fact, spectra up to $\mathrm{X}$ band have become overly crowded with bands for cellular and satellite communications. Therefore, lately investigations on body area networks have been started in the range of millimeter wave frequencies. Millimeter wave frequencies involve signals from $30 \mathrm{GHz}$ to $300 \mathrm{GHz}$. However, BCWC considers the unlicensed spectrum near $\mathrm{V}$ and $\mathrm{W}$ band. Particularly, the V band between 59 and 64 $\mathrm{GHz}$ has recently become a convenient option. Smaller wavelength allowing to design compact body worn sensors and high free space losses reducing interference from adjacent systems are some alluring features of this band. Nevertheless to take the best use of these qualities, characterization of the propagation channels are required. In literature, measurement campaigns have been performed to evaluate path loss distribution [2].Still to arrange the setup for experimental analysis is not always feasible. On the other hand, electromagnetic signal propagation in a Body Area Network creates an intricate problem, analytical methods will provide accurate solutions only in simplified cases [3]. Therefore, numerical simulations, even if computationally more intensive than an analytical approach, can provide a

Dr,Khaleda Ali, EEE,DU physical insight into the propagation mechanism and accurate solutions. Several numerical techniques are available and each of them has its own advantages and drawbacks. In this paper, the prospects of different tools have been discussed and probable solutions have been proposed for accurate analysis.

The rest of the paper is set as below. Section II identifies the most suitable candidate at millimeter wave frequencies for analysis of BAN followed by a brief discussion on some other available simulation tools. Section III focuses on how to customize the appropriate computational technique for the best use of resource and time. The conclusions are presented in Section V.

\section{NUMERICAL TOOLS FOR BAN ANALYSIS}

Potentially a wide variety of modeling methods can be applied to the characterization of BANs. Method of moment, ray based asymptotic methods, Finite Element Method and Finite Difference Time Domain (FDTD) [4] technique are few of them. Next section gives a short introduction of these techniques:

\section{A. Method of Moments}

The method of moments (MoM) technique, introduced by Harrington [5] in electromagnetics, is an extremely efficient computational technique. This method is mostly implemented in the frequency domain. MoM basically deals with solving differential or integral forms of Maxwell's equations numerically. The equations are later transformed into matrix systems of linear equations, where the parameters to be solved designate surface current. At first, these unknown coefficients are expanded in the form of a set of linearly independent known functions (basis functions) multiplied by unknown complex weighting parameters. This method does not necessarily solve Maxwell's equations over the whole simulated structure, which makes this technique extremely popular in dealing with electrically large scatterers.

MoM can be easily implemented to volumes with properties of good conductors $(\sigma \gg \omega \varepsilon)$ or being defined by impedance boundary condition. Rao, Wilton and Glisson have presented a concept of using a set of triangular basis functions to discretize the surface currents over an arbitrary surface [6]. From the calculated surface currents, the total radiated electric field and hence the radiation pattern or the radar cross-section can also be directly derived. MoM was applied to examine the effects of the human body in [4], 
where human subject is approximated as a uniform structured model. Hence, this method is not particularly efficient for investigating on-body propagation scenarios. The classical approach of MoM, with a computational complexity of $\mathrm{O}(\mathrm{N} 2)$ (where $\mathrm{N}$ designates the number of unknowns) is difficult to be parallelised. This is another reason for not considering $\mathrm{MoM}$ as an efficient technique for handling electrically large objects.

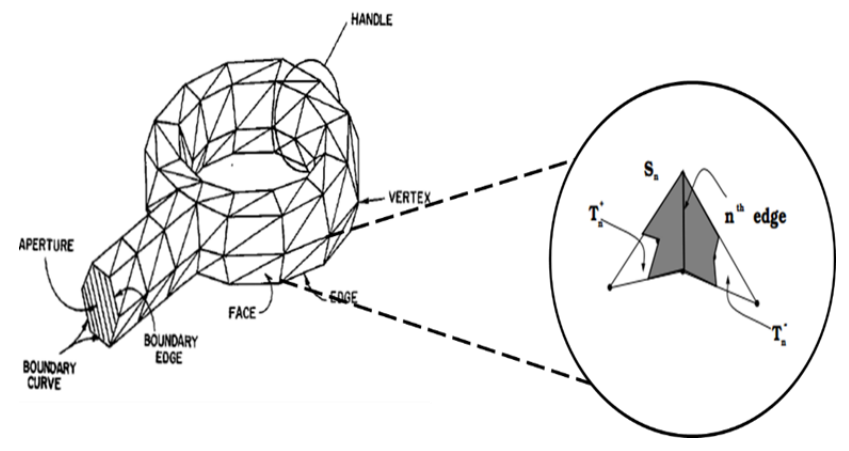

Figure 1: Analysis using Method of moments (reproduced from [5]).

\section{B. Finite Element Method}

The finite element method (FEM) involves dividing complex electromagnetic structures and inhomogeneous medium into a number of elements. This approach is well suited for modelling curved structures and defining them in terms of triangular or rectangular meshes. In addition, it allows variation of the material properties from element to element. The field within each element is expanded in terms of a set of basis polynomials, weighted by the field values at the nodes of the element. Then, the expanded field is substituted into functional form for the Maxwell's equations and the variation of the functional is set to zero. A matrix Eigenvalue equation is derived with the field values at the elements as the unknowns and the solution allows the eigenvalues and eigenvectors to be determined [6].

This formulation exploits the advantages of both the methods and helps in providing an efficient solution for analysing BAN. To decrease the computational burden, it is necessary to reduce the number of elements at the MoM/FEM boundary. To achieve this, a free-space region around the dielectric can be incorporated and discretized using tetrahedral meshes. A coarse discretization can be used for these cells. In spite of the increase in the number of elements in FEM, the decrease in the number of boundary elements helps in optimizing the resources. On the other hand, a hybrid approach combining FEM and MoM can offer flexibilities in performing channel analysis for on-body communication scenario. Commercial software FEKO Suite 5.0 has employed this hybrid approach (as shown in Figure 2). However, this approach is not suitable for high frequency operation of on-body networks when the number of unknowns rises significantly causing a huge increase in the number of cells in FEM domain.

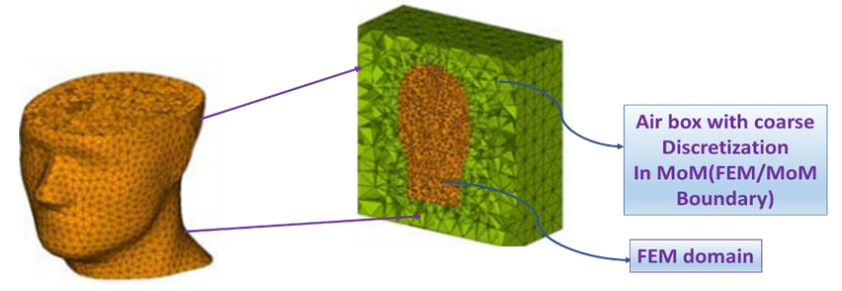

Figure 2. Simulation set up for a Hybrid MoM-FEM approach

\section{Ray based methods}

Ray based asymptotic methods rooted from Fermat's principle [7], such as Geometrical Optics (GO) and Geometrical Theory of Diffraction (GTD) provide efficient solutions for pursuing studies involving electrically larger objects. For higher frequency operation, these methods are more popular than the full wave techniques since they provide less complexity and require limited computational resources (as in Figure 3.).

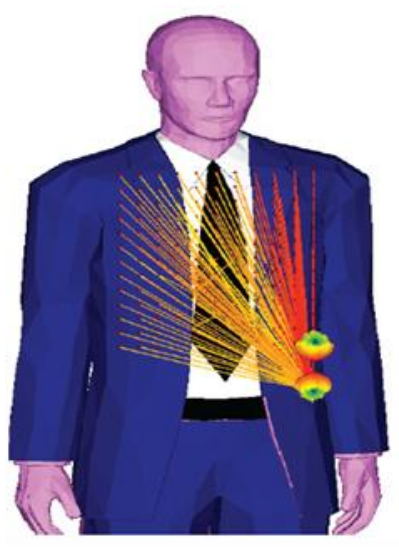

Figure 3. On-body channel analysis using ray-based approach [8]

The Uniform Theory of Diffraction (UTD) is also a popular asymptotic approach. Based on the geometric optics and diffraction theory, this method requires that the objects considered in the simulations to be large compared to the wavelength and assumes all waveforms as locally planar. Direct, reflected and transmitted rays are considered in the UTD domain as in GO. Wedge and corner diffractions following the GTD are also taken into account as they contribute to the total field in the shadow region. Two kinds of ray tracing approaches are available in literature [9]: image method and shooting and bouncing ray method [SBR]. Due to the complexity of propagation scenario for on-body communications, only the SBR approaches are employed. According to this method, the geometrical ray paths are first determined around the subject under investigation. Once the propagation paths are defined, the field strengths are estimated by following the principle of GTD.In addition, the Ray tracing (RT) technique focuses on the site specific prediction for wireless channel characterization. It provides information pertaining to delay of time and angle of incidence for different multipath reception conditions and in the scenario of indoor radio propagation as well. Received signal 
strength and power delay profiles can be correctly predicted using RT techniques provided that the wavelength is small relative to the propagation domain size. Ray-tracing based techniques have been extensively used to predict the propagation channel in indoor and outdoor environments [10]-[12]. In [13], channel variations in Body Area Networks have been presented where all possible ray paths have been taken into account.

A Hybrid of MoM and UTD has also been implemented in solving electromagnetic scattering problems. In such scenarios, accurate MoM is applied to model the source and its nearby objects. Different parameters from MoM solution are later used as equivalent sources and imported in the UTD domain. In [14], indoor radio channel was studied in the presence of human body and the subject was modelled as a conducting circular cylinder. The problem with using UTD and RT is that the human body shape and composition needs to be drastically simplified. Hence, these methods are not suitable for analysis of the on-body radio channel at lower microwave frequencies. However, recent studies have been presented to investigate the prediction accuracy of these asymptotic approaches implemented in on-body communication domain[2]. Commercial software that use a combination of the RT/UTD techniques and suited for higher frequency operation are available. XGTD by Remcom is quite popular among them. This software allows to import three dimensional numerical models composed of facets, are; electromagnetic properties of tissues and fabrics can be defined and the radiation patterns of the antennas can be applied to the transmitter and receiver (as in Figure 3). However XGTD does not take into account transmission through the human body and effects of surface wave along the curvature of the body.

\section{Finite Difference Time Domain Method}

Finite-difference time-domain (FDTD) technique, proposed by Yee in 1966 [15], is based on the direct solution of the time-domain versions of Maxwell's equations. In the domain of computational electromagnetics, it is one of the most suitable techniques for investigating the on-body channel characteristics [16]. The computational complexity of FDTD is $\mathrm{O}(\mathrm{N})$, whereas Method of Moment (MoM) and Finite Element Method (FEM) offer $\mathrm{O}(\mathrm{N})$ complexity. Being a time-domain technique, wideband results can be evaluated from only one simulation using FDTD.

The FDTD is able to handle inhomogeneous mediums very efficiently. Primarily, this numerical tool has been applied in estimating SAR in mobile communications [17]. Extensive study on the effects of different numerical phantoms [18]-[20] in on-body communication scenarios is performed using FDTD. This technique has also been widely used for the investigation of the radiation from implanted devices [21], to evaluate the behavior of body-worn antennas [22], [23]. Frequency-dependent FDTD codes [24]-[26] are developed handling the human body, which is a dispersive and lossy dielectric medium. Literature is available on methods combining FDTD and ray tracing approaches [27], [28] for indoor propagation scenario. However, this does not provide accurate estimation for analyzing communication links near the body. In [29], a new simulation strategy based on the FDTD method has been presented in order to simulate a full-duplex link, considering both the transmitting and receiving antennas. However, the simulation environment does not involve a fine mesh and instead incorporates the concept of bilateral dual-grid-FDTD (as in Figure 4.). Based on this principle, the whole simulation is split into three parts, executed one after another with necessary discretization. The transmitter having finer cell size is initially modelled; rest of the objects are then characterized using a coarse mesh. Finally, the element requiring a high resolution is finely described. This approach is adopted in the investigation of on-body communication at $2.4 \mathrm{GHz}$ and in-body communication at $900 \mathrm{MHz}$. According to [29], this approach provides faster solution compared to conventional algorithms.

millimeter An interesting advantage of the FDTD is the possibility of being parallelized [30]-[32] in order to handle larger electrical problems. Wang et al. have shown in [15] the performance of a PFDTD system (as in Figure 5(a)) in terms of speed and memory resources. Parallel code is extremely useful, particularly in the case of body area networks where human body becomes electrically large. The data transfer functionality between processors (PCs) is provided by the Message Passing Interface (MPI) library. This library is adopted after employing the absorbing boundary conditions in the simulation environment. Data exchange occurs between the adjoining cells at the interface of the sub-domains and is accomplished at every time step [6].

Upon finishing the process, the results evaluated at each sub-domain need to be combined to obtain the overall simulation results. However, as the frequency increases, the huge relative dimensions of the human body become extremely difficult to be modelled. An efficient technique is needed for that purpose. FDTD has the ability to provide accurate solutions of irregularly shaped lossy materials.

Since at the concerned frequency, human body is electrically large relative to the antenna dimension so the concept of uniform theory of diffraction and different ray based techniques are feasible to be applied. But precisely for certain on body links, these approximate procedures lack adequate efficiency. One of the limiting factors of FDTD is the choice of the discretization steps in the computational domain. Increasing step size directly enhances the memory requirements in FDTD. On the other hand, for precise estimation of field distribution, finer cell widths are essential. To obtain a trade-off, an optimized fineness can be selected with a probability of giving better performance. This is a plausible solution even since at millimeter wave frequency signal transmitted through the skin is in range of less than two millimeters and the possible minimum distance between the antenna placed on the body and the upper layer of subject is $1 \mathrm{~mm}$. With this objective a convergence study with different cell size has been conducted in the next section. 


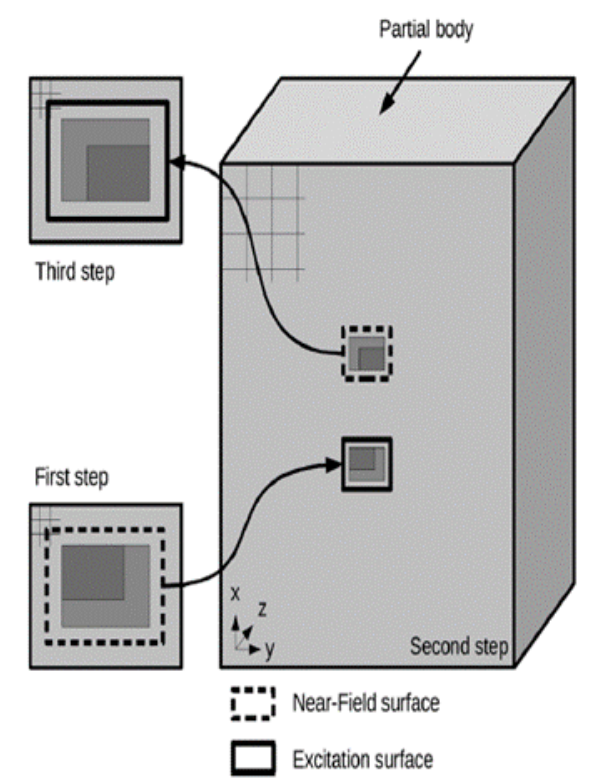

Figure 4. Principle of bilateral dual grid FDTD [29])

\section{NUMERICAL TOOLS FOR BAN ANALYSIS}

To perform the. Convergence study a uniform version of human head is considered.

\section{A. Simulation Setup}

The voxelized model of a human head is shown in Figure 5 (a). In order to consider the curved shape of the human head a smooth structure (Figure 5 (b)) is adopted for the simulation.

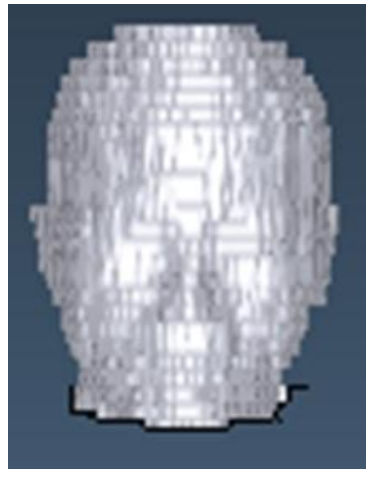

(a)

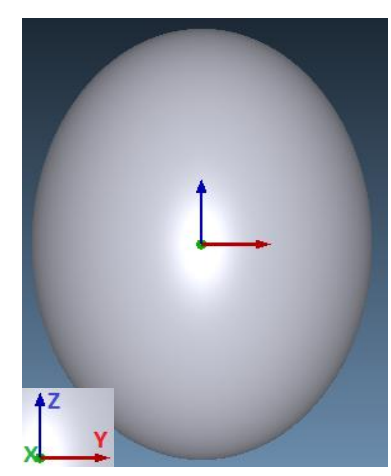

(b)
Figure 5. Numerical model of the human head (a) and structure resembling human head (b)

The whole volume of the structure shown in Figure 5 (b) constitutes of about $18 \times 20 \times 24 \mathrm{~cm} 3$. A 10 cell Berenger's perfect match layer has been considered at the boundary. The dimension in terms of cells varies owing to the variation of discretization step. The electromagnetic properties of the dry skin have been assigned to the model. The dielectric permittivity $\varepsilon$ r and the electric conductivity $\sigma$ at $60 \mathrm{GHz}$ are 7.97 and $36.39 \mathrm{~S} / \mathrm{m}$ [33] respectively. The structure has been split up in several subdomains. A parallel version of FDTD technique has been adopted which analyzes individual parts by separate processors. The source is placed at $2.1 \mathrm{~mm}$ distance from the head. A point source has been applied as the transmitter directed along $\mathrm{x}$ axis (Figure $5(\mathrm{~b})$ ). The subject is discretized from a cell size of $0.3 \mathrm{~mm}-1 \mathrm{~mm}$. A part of the structure has also been simulated considering cell size of $0.1 \mathrm{~mm}$ as it is not possible to simulate the whole structure due to hardware limitations.

\section{B. Results}

The path loss distribution is presented in Figure 6 for 0.35 $\mathrm{mm}$ and $1 \mathrm{~mm}$ discretization step.
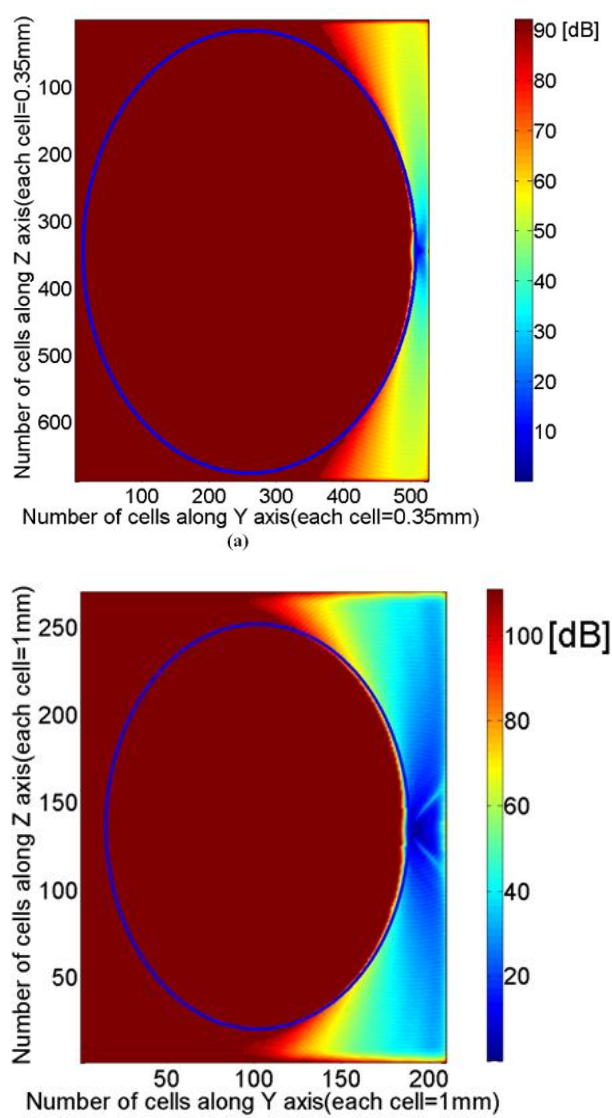

(b)

Figure 6. Path loss distribution on the head (a) With $0.35 \mathrm{~mm}$ cell size (b) With $1 \mathrm{~mm}$ cell size

By observing Figure 6, it is possible to note that the path loss distribution is strongly affected by the cell size. In fact when a cell size of $0.35 \mathrm{~mm}$ (Figure 6-a) is considered, the distribution correctly follows the curvature of the surface, while in the case of a cell size of $1 \mathrm{~mm}$ (Fig. 2-b) the staircase effect is visible. Moreover, it is also evident from the Fig. 2 that the path loss distribution decays rapidly as the distance from the source increases on the head surface. Table I represents the cell size and corresponding computational effort required to complete the simulation.

In order to obtain the path loss distribution around the curvature of the head, a set of observation points, ranging from 0 to 130 degree from the transmitting source for $0.1-1 \mathrm{~mm}$ discretization, are considered and presented in Figure 7. 
TABLE I. PARAMETERS OBTAINED FROM SIMULATION AT DISTINCT CELL SIZE

\begin{tabular}{|c|c|c|c|}
\hline $\begin{array}{c}\text { Cell } \\
\text { Size } \\
(\mathbf{m m})\end{array}$ & $\begin{array}{c}\text { Ratio of } \\
\text { cellsize to } \\
\text { wavelength }\end{array}$ & $\begin{array}{c}\text { Number of } \\
\text { processor } \\
\text { needed to } \\
\text { simulate } \\
\text { whole } \\
\text { structure }\end{array}$ & $\begin{array}{c}\text { Required time } \\
\text { for } \\
\text { processing(hrs) }\end{array}$ \\
\hline 0.1 & $1 / 15$ & 240 & - \\
\hline 0.3 & $1 / 5$ & 83 & 6 \\
\hline 0.35 & $1 / 4$ & 69 & 4 \\
\hline 0.5 & $1 / 3$ & 50 & 2 \\
\hline 1 & $2 / 3$ & 27 & 1 \\
\hline
\end{tabular}

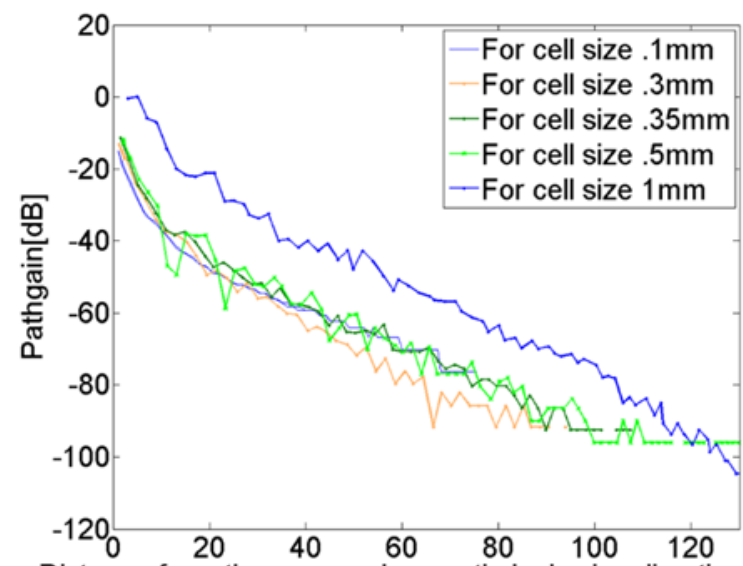

Distance from the source along anti-clock wise direction $(\mathrm{mm})$

Figure 7 Path gain distribution of head for different cell size

It is observed from Figure 7 that the path gain distribution presents a similar trend up to a cell size of $0.5 \mathrm{~mm}$. Therefore, for the specific geometry investigated in this paper, a slightly coarser mesh allow to obtain accurate results in an efficient manner at the same time.

\section{CONCLUSION}

A review has been presented focusing on different numerical techniques available at millimeter wave frequencies. It is evident that FDTD despite having limitations is the best option for channel characterization. To optimize performance with available resources a coarser cell size has been proposed to be used.To this aim a convergence study is accomplished for human head. Parallel version of FDTD technique at $60 \mathrm{GHz}$ has been adopted. Sets of simulation are performed considering different cell sizes. It is observed that for a certain body-source distance cell size can be fixed up to one-third of the wavelength of the constituent material. This occurs since at millimeter wave frequency signal transmitted through the body is significantly low.

\section{REFERENCES}

[1]D. H. Werner and Z. H. Jiang, Electromagnetics of Body-Area Networks: Antennas, Propagation, and RF Systems. 2016.

[2]A. Brizzi, S. Member, A. Pellegrini, and L. Zhang, "Statistical Path-Loss Model for On-Body Communications at $94 \mathrm{GHz}$," vol. 61, no. 11, pp. 5744-5753, 2013.

[3]N. Chahat, G. Valerio, M. Zhadobov, and R. Sauleau, "On-body propagation at $60 \mathrm{GHz}$," IEEE Trans. Antennas Propag., vol. 61, no. 4, pp. 1876-1888, 2013.
[4]F. Keshmiri, S. Member, C. Craeye, and S. Member, "Moment-Method Analysis of Normal-to-Body Antennas Using a Green 's Function Approach," vol. 60, no. 9, pp. 4259-4270, 2012.

[5]S. Rao, D. Wilton, and a. Glisson, "Electromagnetic scattering by surfaces of arbitrary shape," IEEE Trans. Antennas Propag., vol. 30, no. 3, pp. 409-418, May 1982

[6]J. Wang and Q. Wang, Body Area Communications. Singapore: John Wiley \& Sons (Asia) Pte Ltd, 2012.

[7]J. C. Kimball and H. Story, "Fermat's principle, Huygens' principle, Hamilton's optics and sailing strategy," European Journal of Physics, vol. 19 pp. 15-24, 1999.

[8]A. Pellegrini et al., "Antennas and propagation for body-centric wireless communications at millimeter-wave frequencies: A review," IEEE Antennas and Propagation Magazine. 2013.

[9]P. S. Hall and Y. Hao, "Antennas and Propagation for Body-Centric Wireless Communications, Second Edition," in Antennas and Propagation for Body-Centric Wireless Communications, 2012, pp. 1-56,271-355.

[10] G. Tiberi, S. Bertini, W. Q. Malik, A. Monorchio, D. J. Edwards, and G. Manara, "Analysis of Realistic Ultrawideband Indoor Communication Channels by Using an Efficient Ray-Tracing Based Method," IEEE Trans. Antennas Propag., vol. 57, no. 3, pp. 777-785, Mar. 2009.

[11] A. Corucci et al., "A remotely distributed ray tracing for the analysis of electromagnetic propagation in complex indoor and outdoor environments," in 2009 IEEE Antennas and Propagation Society International Symposium, 2009, pp. 1-4.

[12] D. N. Schettino, F. J. S. Moreira, and C. G. Rego, "Efficient Ray Tracing for Radio Channel Characterization of Urban Scenarios," in 2006 12th Biennial IEEE Conference on Electromagnetic Field Computation, 2006, pp. 267-267.

[13] G. Koutitas, "Multiple Human Effects in Body Area Networks," IEEE Antennas Wirel. Propag. Lett., vol. 9, pp. 938-941, 2010.

[14] M. Ghaddar, L. Talbi, T. A. Denidni, and A. Sebak, "A Conducting Cylinder for Modeling Human Body Presence in Indoor Propagation Channel," IEEE Trans. Antennas Propag., vol. 55, no. 11, pp. 3099-3103, Nov. 2007.

[15] J. Wang, O. Fujiwara, S. Watanabe, and Y. Yamanaka, "Computation with a parallel FDTD system of human-body effect on electromagnetic absorption for portable telephones," IEEE Trans. Microw. Theory Tech., vol. 52, pp. 53-58, 2004.

[16] P. S. Hall and Y. Hao, Eds., Antennas and Propagation For Body Centric Wireless Communications, Second. Artech House, 2012.

[17] J. Y. Chen and O. P. Gandhi, "Currents induced in an anatomically based model of a human for exposure to vertically polarized electromagnetic pulses," IEEE Trans. Microw. Theory Tech., vol. 39, pp. 31-39, 1991.

[18] V. Spitzer, M. J. Ackerman, A. L. Scherzinger, and D. Whitlock, "The visible human male: a technical report.," J. Am. Med. Informatics Assoc., vol. 3, pp. 118-130, 1996.

[19] P. J. Dimbylow, "FDTD calculations of the whole-body averaged SAR in an anatomically realistic voxel model of the human body from $1 \mathrm{MHz}$ to 1 GHz.," Phys. Med. Biol., vol. 42, pp. 479-490, 1997.

[20] S. L. Lee, K. Ali, A. Brizzi, J. Keegan, Y. Hao, and G. Z. Yang, "A whole body statistical shape model for radio frequency simulation," in Proceedings of the Annual International Conference of the IEEE Engineering in Medicine and Biology Society, EMBS, 2011, pp. 7143-7146. [21] J. Kim and Y. Rahmat-Samii, "Implanted Antennas Inside a Human Body: Simulations, Designs, and Characterizations," IEEE Trans. Microw. Theory Tech., vol. 52, no. 8, pp. 1934-1943, Aug. 2004.

[22] N. Haga, K. Saito, M. Takahashi, and K. Ito, "Characteristics of cavity slot antenna for body-area networks," IEEE Trans. Antennas Propag., vol. 57, pp. 837-843, 2009.

[23] J. N. Bringuier and R. Mittra, "Electromagnetic wave propagation in Body Area Networks using the Finite-Difference-Time-Domain method.," Sensors (Basel)., vol. 12, no. 7, pp. 9862-83, Jan. 2012.

[24] Y. Zhao, Y. Hao, A. Alomainy, and C. Parini, "UWB on-body radio channel modeling using ray theory and subband FDTD method," in IEEE Transactions on Microwave Theory and Techniques, 2006, vol. 54, pp. 1827-1835.

[25] C. M. Furse and O. P. Gandhi, "The use of the frequency-dependent finite-difference time-domain method for induced current and SAR calculations for a heterogeneous model of the human body," IEEE Trans. Electromagn. Compat., vol. 36, no. 2, pp. 128-133, May 1994.

[26] Q. Wang, T. Tayamachi, I. Kimura, and J. Wang, "An on-body channel model for UWB body area communications for various postures," IEEE Trans. Antennas Propag., vol. 57, pp. 991-998, 2009.

[27] Y. Wang, S. Safavi-Nacini, and S. K. Chaudhuri, "A combined ray tracing and FDTD method for modeling indoor radio wave propagation," IEEE Antennas Propag. Soc. Int. Symp. 1998 Dig. Antennas Gateways to Glob. Netw. Held conjunction with Usn. Natl. Radio Sci. Meet. Cat No98CH36, vol. 3, pp. 1668-1671, 1998. 
[28] M. Yang, S. Stavrou, and A. K. Brown, "Hybrid ray-tracing model for radio wave propagation through periodic building structures," IET Microwaves, Antennas \& Propagation, vol. 5. p. 340, 2011.

[29] C. Miry, R. Loison, and R. Gillard, "An Efficient Bilateral Dual-Grid-FDTD Approach Applied to On-Body Transmission Analysis and Specific Absorption Rate Computation," IEEE Trans. Microw. Theory Tech., vol. 58, no. 9, pp. 2375-2382, Sep. 2010.

[30] A. Taflove and S. C. Hagness, Computational Electrodynamics: The Finite-Difference Time-Domain Method. Artech House, 2000.

[31] Q. H. Abbasi, A. Sani, A. Alomainy, and Y. Hao, "Numerical characterization and modeling of subject-specific ultrawideband body-centric radio channels and systems for healthcare applications.," IEEE Trans. Inf. Technol. Biomed., vol. 16, no. 2, pp. 221-7, Mar. 2012.

[32] Y. Zhao and Y. Hao, "A Subject-Specificity Analysis of Radio Channels in Wireless Body Area Networks," Eng. J., vol. 15, no. 3, pp. 39-48, Jul. 2011.

[33] Italian National Research Council - Institute for Applied Physics, "Dielectric Properties of Body Tissues in the frequency range $10 \mathrm{~Hz}-100$ GHz," INRC, 2012. . 